\title{
Radical cyclisation studies of $\beta$-nitroamines from the nitro-Mannich reaction
}

Abil Aliev, James C. Anderson, ${ }^{*}$ Mérina Corpinot and Emily S. J. Gascoigne

\section{Supplementary Information}

General experimental.

Structural elucidation of $\mathbf{3 1}$ and data correlation table.

Data correlation tables for compounds 34a, 35a, 37, 39, 40a major, 40a minor, 40b major, 40b minor.

X-ray crystallography data for compounds 32a and 35a

General Experimental. All anhydrous chemistry was performed using a Schlenck apparatus and standard syringe techniques; all glassware was flame-dried and reactions were conducted under an inert atmosphere (argon or nitrogen). Room temperature ( $\mathrm{rt}$ ) denotes a value of $20-25^{\circ} \mathrm{C}$, and any temperatures stated reflect the temperature of the external heating/cooling environment. Reaction cooling to $0{ }^{\circ} \mathrm{C}$ was performed in an ice-water bath, to $-20^{\circ} \mathrm{C}$ in an ice-salt bath, to $-40{ }^{\circ} \mathrm{C}$ with a dry ice/acetonitrile bath, and temperatures of $-78^{\circ} \mathrm{C}$ were obtained usually by a dry ice/acetone bath, or a liquid nitrogen/EtOAc bath. Thin layer chromatography was carried out using Polygram ${ }^{\circledR}$ SIL G/UV254 $0.25 \mathrm{~mm}$ silica backed TLC plates, with visualisation by UV light ( $254 \mathrm{~nm}$ ) and $\mathrm{KMnO}_{4}$ or anisaldehyde dip. Flash column chromatography was performed using Gedran ${ }^{\circledR}$ silica gel 60, 40-63 $\mu \mathrm{m}$; the solvent system used is stated in each case. Removal of solvents in vacuo was performed using Büchi rotary evaporators with the house vacuum, diaphragm or Vacuubrand vacuums.

All ${ }^{1} \mathrm{H},{ }^{13} \mathrm{C}$ and ${ }^{19} \mathrm{~F}$ NMR data were obtained using Bruker AMX $300 \mathrm{MHz}$, Bruker AVANCE III $400 \mathrm{MHz}$, Bruker AVANCE $500 \mathrm{MHz}$ or Bruker AVANCE III $600 \mathrm{MHz}$ machines, at room temperature unless stated. Data was manipulated directly using TopSpin (3.2). Unless otherwise stated, NMR data was obtained as a dilute solution in $\mathrm{CDCl}_{3}$, chemical shifts $(\delta)$ are reported in parts per million (ppm) relative to the solvent peak values of $\delta=7.26 \mathrm{ppm}\left({ }^{1} \mathrm{H} \mathrm{NMR}\right)$ and $77.1 \mathrm{ppm}\left({ }^{13} \mathrm{C} \mathrm{NMR}\right)$ for $\mathrm{CDCl}_{3}$. Multiplicities are reported as $\mathrm{s}=$ singlet, $\mathrm{d}=$ doublet, $\mathrm{t}=$ triplet, $\mathrm{q}=$ quartet, quint $=$ quintet, $\mathrm{m}=$ multiplet, br refers to a broad signal, apt = apparent. Coupling constants $(J)$ are measured in Hertz $(\mathrm{Hz})$. Where appropriate, additional NMR techniques have been used to aid assignment (COSY, DEPT, HSQC, HMBC and NOE experiments). Mass spectroscopy analysis data were collected using Thermo Finnigan Mat900xp (EI/CI) or Waters LCT premier XE (ESI) machines. Infrared data were collected on 
a Shimadzu 8700 FT-IR machine as a neat sample (thin film). Elemental analysis was carried out on an Exeter Analytical Inc. EA440 horizontal load analyser. Melting points are reported uncorrected, and were recorded on a Griffin melting point apparatus.

Purification of laboratory reagents. With the exception of specifically mentioned examples, all commercial reagents and solvents detailed were used as supplied or purified by the standard techniques described. ${ }^{269}$ The concentration of commercially-sourced organolithium reagents was verified by titration with $\mathrm{N}$-benzylbenzamide at $-40{ }^{\circ} \mathrm{C} .{ }^{270}$ Dry dichloromethane $\left(\mathrm{CH}_{2} \mathrm{Cl}_{2}\right)$, diethyl ether $\left(\mathrm{Et}_{2} \mathrm{O}\right)$, toluene, tetrahydrofuran (THF) or hexanes were obtained from a solvent tower, in which degassed solvents were passed through two columns of activated alumina and a 7 micron filter under 4 bar pressure. Purification and drying of alcohols, aldehydes, amines or alkenes was carried out via standard procedures, and the resultant purified substances stored under argon at the correct temperature. ${ }^{269}$ Benzene used for radical reactions was purified to remove traces of thiophene as described. ${ }^{269}$ Recrystallisation of AIBN (2,2'-azobis(2-methylproionitrile)) was carried out from $\mathrm{Et}_{2} \mathrm{O}$, the reagent was stored under argon between -5 and $-20^{\circ} \mathrm{C}$. Diiodoethane was washed with sodium thiosulfate solution, dried and stored under argon at $-20^{\circ} \mathrm{C}$. Activation of 3 or 4 Å molecular sieves was performed via heating under high vacuum.

\section{Structural elucidation of 31.}

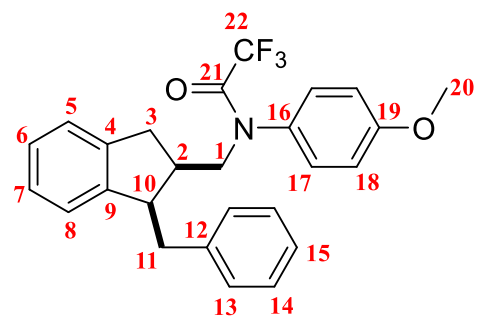

31

Analysis by ${ }^{1} \mathrm{H}$ and ${ }^{13} \mathrm{C}$ NMR techniques confirmed that this was an indane derivative (Full correlation table is given in the appendix). Initial analysis of the molecule was based around the assignment of the $\mathrm{CH}_{2} \mathrm{~N}$ peaks at $\delta 3.74 \mathrm{ppm}(\mathrm{dd}, J=13.3,5.3)$ and $\delta 4.31 \mathrm{ppm}(\mathrm{dd}, J=13.3,9.8)$, which corresponded to a $\mathrm{CH}_{2}$ unit at $\delta 52.0$ ppm by HSQC analysis. Such deshielded peaks for a $\mathrm{CH}_{2}$ unit could be assigned to an electron-poor amine, in this case adjacent to the 2,2,2trifluoroacetamide (\#=1); the shift at $\delta 52.0 \mathrm{ppm}$ was low in comparison with the $C H N$ peak for the initial denitration non-cyclised product $\mathbf{2}$ (Scheme 1, main paper) ( $\delta 59.9 \mathrm{ppm}$ ), due to the loss of the adjacent aromatic ring. Retention of the PMP and TFA groups were confirmed by ${ }^{13} \mathrm{C} \mathrm{NMR}$, with the usual quaternary carbons ( $\delta 131.2 \mathrm{ppm}, \operatorname{ArCN})$ and ( $\delta 159.9 \mathrm{ppm}, \operatorname{ArCO})$ and methyl peak ( $\delta 55.6$ 
ppm, $\mathrm{OCH}_{3}$, also $\delta 3.84 \mathrm{ppm}\left(3 \mathrm{H}, \mathrm{s}, \mathrm{OCH}_{3}\right)$ by $\left.{ }^{1} \mathrm{H} \mathrm{NMR}\right)$ and ${ }^{19} \mathrm{~F} \mathrm{NMR}$, with the expected peak at $\delta$ $66.9 \mathrm{ppm}\left(3 \mathrm{~F}, \mathrm{~s}, \mathrm{CF}_{3}\right)$. It was clear that no aromatic ring had been lost, due to the presence of $9 \mathrm{ArH}$ by ${ }^{1} \mathrm{H}$ NMR, hence it was hypothesised that a rearrangement had occurred, with a proposed structure 31. The proposed structure was investigated by ${ }^{1} \mathrm{H}$ and ${ }^{13} \mathrm{C}$ NMR experiments, including HSQC and HMBC; a NOESY experiment was run to give an idea of the likely stereochemistry across the indane ring. It was known by DEPT and HSQC analysis that $3 \mathrm{CH}_{2}$ units were present in the molecule, at $\delta 35.2,35.4$ and $52.0 \mathrm{ppm}$ by ${ }^{13} \mathrm{C}$ NMR. Ruling out the $\mathrm{CH}_{2} \mathrm{~N}$ peak at $\delta 52.0 \mathrm{ppm}$, carbons 3 and 11 were assigned as either $\delta 35.2$ or 35.4 ppm. The remaining aliphatic $\mathrm{CH}$ peaks at $\delta 41.5$ and $48.0 \mathrm{ppm}$ could be speculatively assigned as carbons 2 and 10 respectively due to their difference in shifts. Carbon 10 ( $\delta 48.0 \mathrm{ppm})$ is adjacent to the aromatic ring of the indane and would likely be shifted further downfield than carbon 2. With a starting point of the $\mathrm{CH}_{2} \mathrm{~N}(\#=1)$ peaks as previously mentioned, the coupling of these protons and the attached carbon to neighbouring protons/carbons was investigated (see correlation table below). It was found that carbon 1 coupled to protons at positions $2(\delta 2.72 \mathrm{ppm}, \mathrm{m})$ and $3(\delta 2.93 \mathrm{ppm}, 2 \mathrm{H}, \mathrm{d}, J=8.2)$, confirming them in close proximity. Protons 2 and 3 were coupled to signals at $\delta 41.5$ and $35.2 \mathrm{ppm}$ in the HSQC spectrum; carbon 10 coupled by HSQC to a proton at $\delta 3.36 \mathrm{ppm}(1 \mathrm{H}, \mathrm{dt}, J=10.2,6.5)$. Carbon 10 also coupled to protons at $1,1^{\prime}, 2,3$, and protons 11 and $11^{\prime}$, which were assigned by this correlation, their benzylic shift (by ${ }^{1} \mathrm{H}$ and ${ }^{13} \mathrm{C}$ NMR of the corresponding carbon at $\left.\delta 35.4 \mathrm{ppm}\right)$, and $\mathrm{HMBC}$ couplings of $\mathrm{C}-11$ to aromatic protons. Carbon 11 and its corresponding protons 11 and $11^{\prime}$ by HSQC were further confirmed by couplings of C-11 to protons 2 and 10; a lack of coupling to protons 1,1' suggested that carbon 11 was the benzylic peak exo to the ring, and not in fact position 3. Carbon 3 is coupled to 1 , $1^{\prime} 5$ and 10 which confirms its closer proximity to $\mathrm{CH}_{2} \mathrm{~N}$ protons $1,1^{\prime}$ and its presence in the indane ring. Further COSY analysis confirms the arrangement of the atoms with couplings between 1-2, 1'-2, 2-3, 2-10, 10-11, and 10-11' as the most diagnostic peaks.

\begin{tabular}{cccccccc}
\hline $\begin{array}{c}\delta_{\mathrm{H}} / \\
\mathbf{p p m}\end{array}$ & Multipl. & $\boldsymbol{J}_{\mathrm{HH}} / \mathrm{Hz}$ & $\#$ & COSY & $\begin{array}{c}\delta_{\mathrm{c}} / \\
\mathrm{ppm}\end{array}$ & HMBCs & NOEs \\
\hline $\mathbf{2 . 6 1}$ & $\mathrm{dd}$ & $13.3,10.3$ & $\mathbf{1 1 ^ { \prime }}$ & 10,11 & 35.4 & - & \\
$\mathbf{2 . 7 2}$ & $\mathrm{m}$ & - & $\mathbf{2}$ & $1,1^{\prime}, 3$ & 41.5 & $1,1^{\prime}, 10,3,11,11^{\prime}$ & $\mathbf{1 0}(\mathrm{s})$ \\
$\mathbf{2 . 9 0}$ & $\mathrm{dd}$ & $13.4,6.0$ & $\mathbf{1 1}$ & $10,11^{\prime}$ & 35.4 & $13,2,10$ & \\
$\mathbf{2 . 9 3}$ & $2 \mathrm{H}, \mathrm{d}$ & 8.2 & $\mathbf{3}$ & 2 & 35.2 & $5,1,1^{\prime},(10$ & \\
$\mathbf{3 . 3 6}$ & $\mathrm{dt}$ & $10.2,6.5$ & $\mathbf{1 0}$ & 2,11, & 48.0 & $8,1,1^{\prime}, 3,11,11^{\prime}, 2$ & $\mathbf{2}(\mathrm{s})$ \\
& & & & $11^{\prime}$ & & & \\
\hline
\end{tabular}




\begin{tabular}{|c|c|c|c|c|c|c|}
\hline 3.74 & dd & $13.3,5.3$ & $1^{\prime}$ & 1,2 & 52.0 & - \\
\hline 3.84 & s & & 20 & & 55.6 & - \\
\hline 4.31 & dd & $13.3,9.8$ & 1 & $1^{\prime}, 2$ & 52.0 & 3,2 \\
\hline 6.42 & $d$ & 7.5 & 8 & & 125.0 & 6 \\
\hline $\begin{array}{c}6.87- \\
6.92\end{array}$ & $\mathrm{~m}$ & & 18 & & $\begin{array}{c}\sim 115.0 \\
\text { br }\end{array}$ & \\
\hline 6.94 & $\mathrm{td}$ & $7.5,1.0$ & 7 & & 125.8 & 5 \\
\hline $\begin{array}{c}6.96- \\
7.00\end{array}$ & $\mathrm{~m}$ & & 13 & & 129.4 & $3,3^{\prime}$ \\
\hline 7.13 & $\mathrm{td}$ & $7.5,0.9$ & 6 & & 126.9 & 8 \\
\hline 7.15 & $\mathrm{~m}$ & & 17 & & $\sim 130 \mathrm{br}$ & \\
\hline- & $\mathrm{m}$ & & 15 & & 126.2 & 130 \\
\hline \multirow[t]{2}{*}{7.25} & $d$ & 7.5 & 5 & & 124.7 & 3 \\
\hline & $\mathrm{m}$ & & 14 & & 128.3 & \\
\hline- & & $\begin{array}{c}{ }^{1} \mathrm{~J}_{\mathrm{CF}}= \\
288.6\end{array}$ & 22 & & 116.6 & \\
\hline- & & & 16 & & 131.2 & $17,18,1,1^{\prime}$ \\
\hline- & & & 12 & & 139.8 & $14,10,11,11^{\prime}$ \\
\hline- & & & 4 & & 141.8 & $6,8,10,3$ \\
\hline- & & & 9 & & 145.6 & $5,7,10,3,11,11^{\prime}$ \\
\hline \multirow[t]{2}{*}{-} & & ${ }^{2} J_{C F}=$ & 21 & & 157.5 & $1,1^{\prime}$ \\
\hline & & 34.9 & & & & \\
\hline- & & & 19 & & 159.9 & $17,18,20$ \\
\hline
\end{tabular}

\section{Structural elucidation of 32a and 32a'.}

Full characterisation of both compounds was undertaken, with confirmation of the molecular ion peaks at $498\left(\mathrm{M}+\mathrm{H}^{+}\right)$and $497\left(\mathrm{M}^{+}\right)$by MS analysis of 32a and 32a'. The indane ring in both products 
was confirmed as for $\mathbf{3 1}$, using ${ }^{1} \mathrm{H} N M R$ and ${ }^{13} \mathrm{C}$ techniques, $\mathrm{HSQC}$ and $\mathrm{HMBC}$ providing the structural information necessary to assign relative stereochemistry.

\section{Data correlation table for $34 a$.}

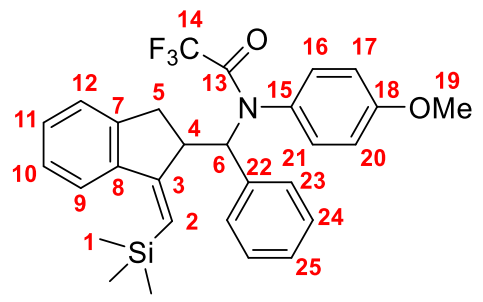

$34 a$

\begin{tabular}{|c|c|c|c|c|c|c|c|}
\hline$\delta_{\mathrm{H}} / \mathrm{ppm}$ & Mult. & $\mathrm{J}_{\mathrm{HH}} / \mathrm{Hz}$ & $\#$ & COSY & $\delta_{c} / \mathrm{ppm}$ & $H M B C S$ & NOESY \\
\hline 0.29 & $9 \mathrm{H}, \mathrm{s}$ & & 1 & & 0.05 & & 9 \\
\hline 2.27 & $\begin{array}{c}1 \mathrm{H}, \text { apt } \\
\mathrm{d}\end{array}$ & 16.7 & 5 & 4 & 35.5 & $\begin{array}{c}3,4,6,7 \\
8,12\end{array}$ & \\
\hline 3.04 & $1 \mathrm{H}, \mathrm{dd}$ & $\begin{array}{c}16.6 \\
7.5\end{array}$ & $5^{\prime}$ & 4,12 & 35.5 & $4,6,7,8$ & \\
\hline 3.78 & $3 \mathrm{H}, \mathrm{s}$ & & 19 & & 55.5 & 18 & \\
\hline 4.21 & $\begin{array}{c}1 \mathrm{H}, \mathrm{br} \\
\mathrm{dd}\end{array}$ & $\begin{array}{c}10.8 \\
7.5\end{array}$ & 4 & $5^{\prime}, 6$ & 48.0 & $5,5^{\prime}, 6,7$ & 2 \\
\hline 4.55 & $1 \mathrm{H}, \mathrm{brd}$ & 10.8 & 6 & 4 & $71.1(\mathrm{br})$ & $4,5,5^{\prime}$ & \\
\hline 5.99 & $1 \mathrm{H}, \mathrm{s}$ & & 2 & 4 & 124.8 & $\begin{array}{c}1,2,4,6 \\
8\end{array}$ & $\begin{array}{l}\text { 4, } \\
\text { PMP }\end{array}$ \\
\hline 6.67 & $1 \mathrm{H}, \mathrm{dd}$ & $8.9,3.0$ & PMP & & 114.3 & & \\
\hline $6.71-6.74$ & $1 \mathrm{H}, \mathrm{m}$ & & PMP & & 131.2 & & 2 \\
\hline 6.80 & $1 \mathrm{H}, \mathrm{dd}$ & $8.7,2.7$ & PMP & & 113.5 & & \\
\hline 7.07 & $1 \mathrm{H}, \mathrm{m}$ & & PMP & & 130.1 & & 2 \\
\hline 7.11 & $1 \mathrm{H}, \mathrm{d}$ & 7.5 & 12 & $5^{\prime}$ & 125.7 & 5,8 & \\
\hline $7.18-7.31$ & $7 \mathrm{H}, \mathrm{m}$ & & ArH & & & & \\
\hline
\end{tabular}




\begin{tabular}{|c|c|c|c|c|c|c|}
\hline \multirow[t]{8}{*}{7.67} & $1 \mathrm{H}, \mathrm{d}$ & 7.7 & 9 & 124.6 & 2,7 & 1 \\
\hline & & & 15 & 130.4 & & \\
\hline & & & 22 & 137.3 & & \\
\hline & & & 8 & 140.4 & $2,5,5^{\prime}, 12$ & \\
\hline & & & 7 & 145.7 & $4,5,5^{\prime}, 9$ & \\
\hline & & 35.5 & 14 & 156.9 & & \\
\hline & & & 3 & 159.3 & $2,5,9$ & \\
\hline & & & 18 & 159.6 & 19 & \\
\hline
\end{tabular}

Key NOESY interactions to confirm geometry of alkene.

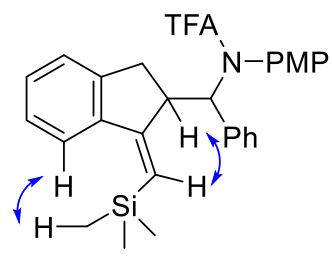

Data correlation table for 35a.

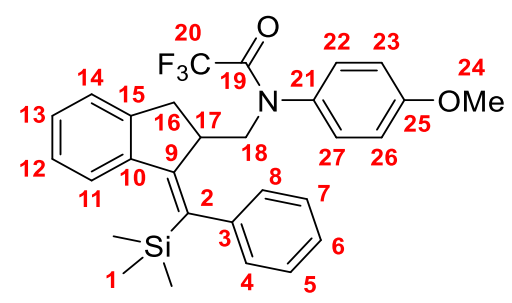

$35 a$

\begin{tabular}{|c|c|c|c|c|c|}
\hline$\delta_{\mathrm{H}} / \mathrm{ppm}$ & Mult. & $J_{\mathrm{HH}} / \mathrm{Hz}$ & $\#$ & $\delta \mathrm{c} / \mathrm{ppm}$ & HMBCS \\
\hline 0.07 & $9 \mathrm{H}, \mathrm{s}$ & & 1 & 0.5 & 1 \\
\hline 2.72 & $1 \mathrm{H}$, apt d & 15.4 & 16 & 33.5 & $13,14,15,17,18$ \\
\hline 2.90 & $1 \mathrm{H}, \mathrm{dd}$ & $13.3,3.3$ & 18 & 52.3 & 16,17 \\
\hline $2.93-3.03$ & $2 \mathrm{H}, \mathrm{m}$ & & $\begin{array}{l}16 \& \\
17\end{array}$ & $33.5 \& 42.1$ & $\begin{array}{l}16(12,13,15,17 \text {, } \\
18) \mathbf{1 7} \text { (qs) }\end{array}$ \\
\hline 3.85 & $3 \mathrm{H}, \mathrm{s}$ & & 24 & 55.6 & (qs) \\
\hline 4.01 & $1 \mathrm{H}, \mathrm{dd}$ & $13.1,11.5$ & 18 & 52.3 & 16,17 \\
\hline
\end{tabular}




\begin{tabular}{|c|c|c|c|c|}
\hline $6.60-7.17$ & $9 \mathrm{H}, \mathrm{br} \mathrm{m}$ & & & \\
\hline 7.24 & $1 \mathrm{H}$, apt t & 12 & 126.0 & \\
\hline 7.28 & $1 \mathrm{H}, \mathrm{dd}$ & 13 & 128.8 & \\
\hline 7.32 & $1, d$ & 14 & 126.3 & 15 \\
\hline \multirow[t]{9}{*}{7.66} & $1 \mathrm{H}, \mathrm{d}$ & 11 & 125.5 & 15 \\
\hline & & 21 & 130.0 & 18 \\
\hline & & 2 & 139.4 & 1,17 \\
\hline & & 15 & 139.8 & $11,14,16$ \\
\hline & & 3 & 145.2 & (Ar) \\
\hline & & 10 & 146.6 & $11,16,17$ \\
\hline & & 9 & 153.5 & $11,16,17,18$ \\
\hline & & 19 & 157.6 & 18 \\
\hline & & 25 & 159.3 & 24 \\
\hline
\end{tabular}

Data correlation table for 37.

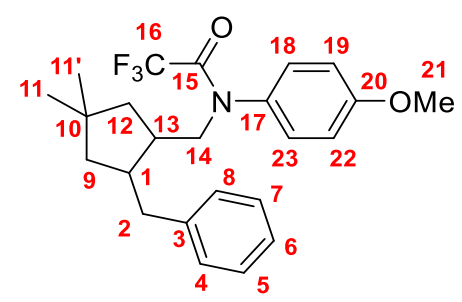

37

\begin{tabular}{cccccc}
\hline$\delta_{\mathrm{H}} / \mathrm{ppm}$ & Mult. & $J_{\mathrm{HH}} / \mathrm{Hz}$ & $\#$ & COSY & $\delta_{\mathrm{c}} / \mathrm{ppm}$ \\
\hline $\mathbf{0 . 9 2}$ & $3 \mathrm{H}, \mathrm{s}$ & - & $\mathbf{1 1}$ & 30.6 \\
$\mathbf{1 . 1 1}$ & $3 \mathrm{H}, \mathrm{s}$ & - & $11^{\prime}$ & 31.4 \\
$\mathbf{1 . 3 4}$ & $1 \mathrm{H}, \mathrm{dd}$ & $13.0,8.5$ & $\mathbf{9}$ & 46.3 \\
$\mathbf{1 . 4 0}$ & $1 \mathrm{H}, \mathrm{dd}$ & $13.0,6.6$ & $\mathbf{9}$ & 46.3 \\
$\mathbf{1 . 5 0}$ & $1 \mathrm{H}, \mathrm{dd}$ & $13.0,7.8$ & $\mathbf{1 2}$ & 44.7 \\
$\mathbf{1 . 5 8}$ & $1 \mathrm{H}, \mathrm{dd}$ & $13.0,7.3$ & $\mathbf{1 2}$ & 44.7 \\
\hline
\end{tabular}




\begin{tabular}{|c|c|c|c|c|c|}
\hline 2.27 & $1 \mathrm{H}, \mathrm{m}$ & & 13 & 12,14 & 38.8 \\
\hline 2.38 & $1 \mathrm{H}, \mathrm{m}$ & & 1 & 9,13 & 42.4 \\
\hline 2.43 & $1 \mathrm{H}, \mathrm{dd}$ & $12.8,10.7$ & 2 & $4 / 8$ & 36.5 \\
\hline 2.70 & $1 \mathrm{H}, \mathrm{dd}$ & $12.8,4.7$ & 2 & $4 / 8$ & 36.5 \\
\hline 3.49 & $1 \mathrm{H}, \mathrm{dd}$ & $13.1,4.9$ & 14 & 13 & 52.4 \\
\hline 3.85 & $3 \mathrm{H}, \mathrm{s}$ & - & 21 & & 55.6 \\
\hline 4.25 & $1 \mathrm{H}, \mathrm{dd}$ & $13.0,11.0$ & 14 & & 52.4 \\
\hline 6.91 & $2 \mathrm{H}$, apt d & 8.7 & $19 \& 22$ & & $114.0,114.7$ \\
\hline 7.08 & $2 \mathrm{H}$, apt d & 7.8 & $4 \& 8$ & & 128.8 \\
\hline 7.11 & $2 \mathrm{H}, \mathrm{d}$ & 8.6 & $18 \& 23$ & & $129.4,130.0$ \\
\hline 7.16 & $1 \mathrm{H}, \mathrm{tm}$ & 7.4 & 6 & & 125.9 \\
\hline \multirow[t]{2}{*}{$7.22-7.25$} & $2 \mathrm{H}, \mathrm{m}$ & - & $5 \& 7$ & & 128.4 \\
\hline & & & 10 & & 37.4 \\
\hline
\end{tabular}

Data correlation table for 39.

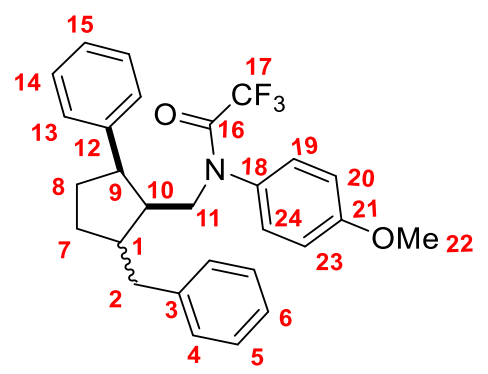

39

\begin{tabular}{|c|c|c|c|c|c|c|c|}
\hline $\begin{array}{c}\delta_{\mathrm{H}} / \\
\mathrm{ppm}\end{array}$ & Mult. & $\begin{array}{c}J_{\mathrm{HH}} / \\
\mathrm{Hz}\end{array}$ & $\#$ & $\cos Y$ & $\delta_{c} / \mathrm{ppm}$ & $H M B C S$ & NOESY \\
\hline 1.54 & $\mathrm{~m}$ & - & 7 & $7^{\prime}, 1 / 2$ & 30.9 & & \\
\hline 1.61 & $1 \mathrm{H}, \mathrm{m}$ & - & 8 & $8^{\prime}$ & 34.8 & & \\
\hline 1.74 & $1 \mathrm{H}, \mathrm{m}$ & - & $7^{\prime}$ & $7,8^{\prime}, 1$ & 30.9 & & \\
\hline $\begin{array}{c}2.25- \\
2.33\end{array}$ & $2 \mathrm{H}, \mathrm{m}$ & - & 8' \& & $\left(7,8^{\prime}, 7^{\prime}\right)$ & 34.8 & & \\
\hline
\end{tabular}




\begin{tabular}{|c|c|c|c|c|c|c|c|}
\hline & & & 10 & $(11,8,9)$ & 47.6 & & $1(\mathrm{w}) \boldsymbol{9}(\mathrm{s})$ \\
\hline \multirow[t]{2}{*}{$\begin{array}{c}2.40- \\
2.47\end{array}$} & $2 \mathrm{H}, \mathrm{m}$ & - & $2 \&$ & 7 & 35.7, & & \\
\hline & & & 1 & & 44.1 & & $9(w) 10(w)$ \\
\hline 2.77 & $1 \mathrm{H}, \mathrm{m}$ & - & $2^{\prime}$ & $1 / 2$ & 35.7 & & \\
\hline \multirow[t]{2}{*}{3.13} & $1 \mathrm{H}$, apt & 8.4 & 9 & 10 & 49.1 & & $10(\mathrm{~s}) 1(\mathrm{w})$ \\
\hline & $q$ & & & & & & \\
\hline 3.62 & $1 \mathrm{H}, \mathrm{dd}$ & $\begin{array}{c}13.5 \\
6.0\end{array}$ & 11 & 10 & 52.7 & & \\
\hline 3.79 & $3 \mathrm{H}, \mathrm{s}$ & - & 22 & & & & \\
\hline \multirow[t]{6}{*}{4.28} & $1 \mathrm{H}, \mathrm{dd}$ & 13.4, & 11 & 10 & 52.7 & & \\
\hline & & & 18 & & $131.0(q)$ & 11 & \\
\hline & & & 3 & & $141.2(q)$ & 2 & \\
\hline & & & 12 & & $146.6(q)$ & 9 & \\
\hline & & & 16 & & $157.6(q)$ & 11 & \\
\hline & & & 21 & & 159.5 (q) & 22 & \\
\hline
\end{tabular}

Data correlation table for 40 a major.

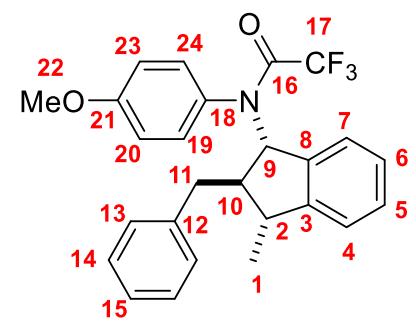

40a major

\begin{tabular}{cccccccc}
\hline $\begin{array}{c}\delta_{\mathrm{H}} / \\
\text { ppm }\end{array}$ & Mult. & $J_{\mathrm{HH}} / \mathrm{Hz}$ & $\#$ & COSY & $\begin{array}{c}\delta_{\mathrm{c}} / \\
\text { ppm }\end{array}$ & HMBCS & NOESY \\
\hline $\mathbf{0 . 9 7}$ & $3 \mathrm{H}, \mathrm{d}$ & 7.0 & $\mathbf{1}$ & $2,10(\mathrm{w})$ & 18.8 & $2,9(\mathrm{w}), 10$ & \\
$\mathbf{1 . 9 9}$ & $\mathrm{tdd}$ & $8.7,7.6,6.1$ & $\mathbf{1 0}$ & $\begin{array}{c}1(\mathrm{w}), 2,9, \\
11^{\prime}\end{array}$ & 51.4 & $1,2,11,11^{\prime}$, & $\mathbf{2 ( - )}$ \\
& & & & & 9 & $\mathbf{1 1}(\mathrm{m})$ \\
\hline
\end{tabular}




\begin{tabular}{|c|c|c|c|c|c|c|c|}
\hline 2.85 & $\mathrm{~m}$ & - & 2 & 1,10 & 42.6 & $\begin{array}{c}1,10,11,11^{\prime} \\
\& A r H\end{array}$ & \\
\hline 2.95 & dd & $13.7,6.0$ & 11 & $10,11^{\prime}$ & 39.2 & $2,9,10, \mathrm{ArH}$ & \\
\hline 3.03 & dd & $13.7,7.5$ & $11^{\prime}$ & 10,11 & 39.2 & $2,9,10, \mathrm{ArH}$ & \\
\hline 3.76 & $3 \mathrm{H}, \mathrm{s}$ & & 22 & & 55.4 & - & \\
\hline 6.28 & $d$ & 8.8 & 9 & 2,10 & 67.0 & $10,11,11^{\prime}$ & $\begin{array}{c}2(\mathrm{w}) \\
10(\mathrm{~m}) \\
11(\mathrm{~m})\end{array}$ \\
\hline 6.37 & Apt d & 8.6 & PMP & & $\begin{array}{c}113.6 \\
-\end{array}$ & - & \\
\hline 6.59 & dd & $8.9,2.9$ & PMP & & 132.1 & & \\
\hline $\begin{array}{l}6.61- \\
6.65\end{array}$ & $\mathrm{~m}$ & - & PMP & & & & \\
\hline 6.76 & $d d$ & $8.8,2.9$ & PMP & & & & \\
\hline $\begin{array}{l}7.09- \\
7.13\end{array}$ & $2 \mathrm{H}, \mathrm{m}$ & - & ArH & & & & \\
\hline \multirow[t]{10}{*}{$\begin{array}{l}7.23- \\
7.39\end{array}$} & $7 \mathrm{H}, \mathrm{m}$ & - & ArH & & & & \\
\hline & & ${ }^{1} J_{C F}=$ & 17 & & 116.6 & - & \\
\hline & & 288.3 & & & & & \\
\hline & & & 18 & & 127.9 & 9, PMP & \\
\hline & & & 8 & & 139.6 & $2,9,10$ & \\
\hline & & & 12 & & 139.6 & $10,11,11^{\prime}$ & \\
\hline & & & 3 & & 146.7 & $1,2,9, \mathrm{ArH}$ & \\
\hline & & ${ }^{2} J_{C F}=$ & 16 & & 158.2 & & \\
\hline & & 35.1 & & & & & \\
\hline & & & 21 & & 159.8 & & \\
\hline
\end{tabular}


Data correlation table for $40 a$ minor.

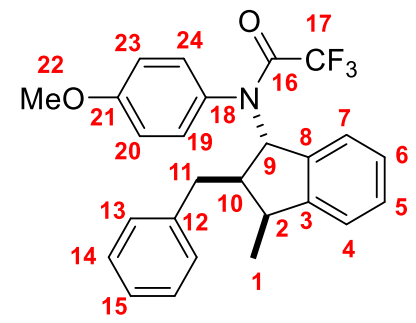

40a minor

\begin{tabular}{|c|c|c|c|c|c|c|c|}
\hline $\begin{array}{l}\delta_{H} / \\
p p m\end{array}$ & Mult. & $J_{H H} / H z$ & $\#$ & $\cos Y$ & $\begin{array}{l}\delta_{c} / \\
p p m\end{array}$ & HMBCS & NOESY \\
\hline 1.12 & $3 \mathrm{H}, \mathrm{d}$ & 7.3 & 1 & $\begin{array}{l}\left(2 / 11^{\prime}\right)^{a} 9 \\
(w)\end{array}$ & 17.3 & $\begin{array}{l}\left(2 / 11^{\prime}\right)^{a}, \\
10\end{array}$ & \\
\hline 2.60 & tdd & $\begin{array}{l}9.2,7.9, \\
6.7\end{array}$ & 10 & $\left(2 / 11^{\prime}\right)^{a}, 9$ & 39.3 & 1,11 & $\begin{array}{l}2(s) 9 \\
(w) 11 \\
(-)\end{array}$ \\
\hline
\end{tabular}

$\begin{array}{lllllll}2.90 & d d & 14.5,9.2 & 11 & 10 & 34.4 & 1,\end{array}$

$\left(2 / 11^{\prime}\right)^{a}$,

9,10

2.96- $2 \mathrm{H}, \mathrm{m}$

$11^{\prime} \& \quad 1,10$

$34.4 \&$

3.04

2

$45.3 \quad 1$,

$\left(2 / 11^{\prime}\right)^{a}$,

9,11

$3.77 \quad 3 \mathrm{H}, \mathrm{s}$

22

55.5

$6.24 d$

9.2

$9 \quad 10, \mathrm{ArH}$

66.3

$\begin{array}{ll}\left(2 / 11^{\prime}\right)^{\mathrm{a}}, & \mathbf{1 0}(\mathrm{w}) \\ 10,11, & \mathbf{2 ( - )} \mathbf{1 1} \\ \text { ArH } & \text { (m) }\end{array}$

6.61- m

PMP

113.6 -

6.65

132.1
6.70
$\mathrm{dm}$
8.7
PMP 


\begin{tabular}{|c|c|c|c|c|c|}
\hline 6.83 & $\mathrm{~m}$ & & PMP & & \\
\hline 7.18 & $2 \mathrm{H}$, apt d & 7.5 & ArH & & \\
\hline \multirow{11}{*}{$\begin{array}{l}7.23- \\
7.39\end{array}$} & $7 \mathrm{H}, \mathrm{m}$ & & ArH & & \\
\hline & & ${ }^{1} J_{C F}=$ & 17 & 116.6 & \\
\hline & & 289.2 & & & \\
\hline & & & 18 & 127.8 & 9, PMP \\
\hline & & & 8 & 139.1 & 9, ArH \\
\hline & & & 12 & 140.0 & 10,11 \\
\hline & & & & & $11^{\prime}, \mathrm{ArH}$ \\
\hline & & & 3 & 148.7 & 1, 2/11', \\
\hline & & & & & 9, $\mathrm{ArH}$ \\
\hline & & ${ }^{2} J_{C F}=34.6$ & 16 & 158.5 & 9 \\
\hline & & & 21 & 160.0 & PMP \\
\hline
\end{tabular}

${ }^{a}$ Coincident signals

\section{Structural elucidation of diastereoisomers $40 \mathrm{~b}$}

Assignment of the major and minor diastereoisomers was conducted by ${ }^{1} \mathrm{H} N M R$, using ${ }^{3}{ }_{\mathrm{HH}}$ coupling values for the indane ring protons and NOESY interactions. Analysis of $J$ couplings showed that for the major diastereoisomer ${ }^{3} J_{1,2}=7.1 \mathrm{~Hz},{ }^{3} J_{2,3}=\sim 7 \mathrm{~Hz}$, suggesting a common orientation of protons $\mathrm{H}_{1}-\mathrm{H}_{2}$ to $\mathrm{H}_{2}-\mathrm{H}_{3}$ in the cis-region as previously verified for 32a. However NOESY correlations showed $\mathrm{H}_{1}-\mathrm{H}_{2}(w), \mathrm{H}_{2}-\mathrm{H}_{3}(w)$ and $\mathrm{H}_{1}-\mathrm{H}_{3}(m)$, suggesting a trans, trans-configuration. This diastereoisomer demonstrates the difficulty in assignment of indanes, and the requirement for multi-faceted evidence for assignment. In comparison the minor isomer was tentatively assigned as the trans,cisisomer, with a larger coupling constant for ${ }^{3} J_{1,2}=9.2 \mathrm{~Hz}$, which more convincingly suggested a transorientation of these protons, further corroborated by a weak NOESY interaction. The orientation of protons $\mathrm{H}_{2}-\mathrm{H}_{3}$ was assigned as cis- due to the strong NOESY interaction between them, a coupling constant ${ }^{3} J_{2,3}=7.5 \mathrm{~Hz}$ and also a lack of interaction between protons $\mathrm{H}_{1}-\mathrm{H}_{3}$. The $\mathrm{CHN}{ }^{1} \mathrm{H}$ NMR shifts of both protons $\mathrm{H}_{1}$ in $40 \mathrm{~b}$ were also very similar to each other $(\delta 6.31 \mathrm{ppm}$ major and $6.28 \mathrm{ppm}$ minor) and to those of $40 \mathrm{a}(\delta 6.28 \mathrm{ppm}$ and $6.24 \mathrm{ppm})$ suggesting little change in environment between $\mathrm{H}_{1}-\mathrm{H}_{2}$ in all four molecules (trans-).

Data correlation table for $40 \mathrm{~b}$ major. 


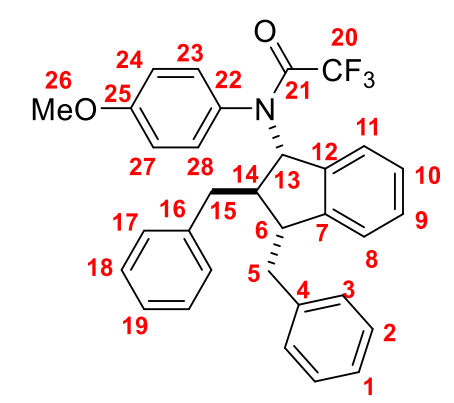

40b major

\begin{tabular}{|c|c|c|c|c|c|c|}
\hline$\delta_{H} / p p m$ & Mult. & $J_{H H} / H z$ & $\#$ & $\begin{array}{l}\delta_{c} / \\
p p m\end{array}$ & HMBCS & NOESY \\
\hline 2.21 & $1 \mathrm{H}, \mathrm{dtd}$ & 9.6, 7.1, 4.8, & 14 & 47.5 & & $13(w)$ \\
\hline 2.24 & $1 \mathrm{H}, \mathrm{dd}$ & $13.4,8.2$ & 5 & 41.2 & & \\
\hline 2.51 & $1 \mathrm{H}, \mathrm{dd}$ & $13.3,4.6$ & 15 & 41.0 & & \\
\hline 2.70 & $1 \mathrm{H}, \mathrm{dd}$ & $13.4,9.6$ & $15^{\prime}$ & 41.0 & & \\
\hline 2.79 & $1 \mathrm{H}, \mathrm{dd}$ & $13.6,6.3$ & $5^{\prime}$ & 41.2 & & \\
\hline \multirow[t]{2}{*}{$3.13-3.18$} & $\mathrm{~m}$ & & 6 & 49.8 & & $13(\mathrm{~m})$ \\
\hline & & & & & & $14(w)$ \\
\hline 3.78 & $3 \mathrm{H}, \mathrm{s}$ & - & 26 & 55.5 & & \\
\hline 6.17 & $1 \mathrm{~h}, \mathrm{~d}$ & 7.8 & PMP & & & \\
\hline 6.31 & $1 \mathrm{H}, \mathrm{d}$ & 7.1 & 13 & 67.3 & & \\
\hline 6.42 & $1 \mathrm{H}, \mathrm{dd}$ & $8.7,2.6$ & PMP & & & \\
\hline 6.54 & $1 \mathrm{H}, \mathrm{dd}$ & $8.9,3.0$ & PMP & & & \\
\hline 6.75 & $1 \mathrm{H}, \mathrm{dd}$ & $8.8,3.0$ & PMP & & & \\
\hline 6.91 & $2 \mathrm{H}, \mathrm{m}$ & - & $\mathrm{Ar}$ & & & \\
\hline 7.05 & $\begin{array}{l}3 \mathrm{H}, \text { apt } \\
\mathrm{d}\end{array}$ & 7.0 & $\mathrm{Ar}$ & & 12 & \\
\hline $7.15-7.35$ & $8 \mathrm{H}, \mathrm{m}$ & - & $\mathrm{Ar}$ & & 12,7 & \\
\hline \multirow[t]{3}{*}{7.37} & $1 \mathrm{H}, \mathrm{d}$ & 7.4 & $\mathrm{Ar}$ & & 7 & \\
\hline & & 288.7 & 20 & 116.6 & & \\
\hline & & & 22 & 128.0 & PMP & \\
\hline
\end{tabular}




\begin{tabular}{llll}
\hline $\mathbf{4}$ & 139.3 & 5 \\
& $\mathbf{1 6}$ & 139.6 & 15 \\
& $\mathbf{1 2}$ & 140.2 & 13 \\
& $\mathbf{7}$ & 145.6 & $5,6,13$ \\
& $\mathbf{2 1}$ & 157.6 & \\
& $\mathbf{2 5}$ & 159.8 & \\
\hline
\end{tabular}

Data correlation table for $40 \mathrm{~b}$ minor.

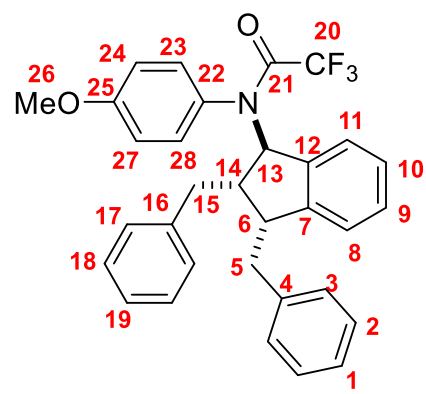

40b minor

\begin{tabular}{llllllll}
\hline $\begin{array}{l}\delta_{H} / \\
\text { ppm }\end{array}$ & Mult. & $J_{H H} / \mathrm{Hz}$ & $\#$ & COSY & $\begin{array}{l}\delta_{c} / \\
\text { ppm }\end{array}$ & HMBCS & NOESY \\
\hline $\mathbf{2 . 4 4}$ & $1 \mathrm{H}, \mathrm{dd}$ & $13.4,8.2$ & $\mathbf{5}$ & & 36.5 & 6,14 & $13(\mathrm{~m})$
\end{tabular}




\begin{tabular}{|c|c|c|c|c|c|c|c|}
\hline 2.69 & $1 \mathrm{H}, \mathrm{m}$ & & 14 & $6,13,15$ & 46.0 & $\begin{array}{l}5^{\prime}, 6,13, \\
15\end{array}$ & $6(\mathrm{~s})$ \\
\hline 3.05 & $2 \mathrm{H}, \mathrm{m}$ & & 15 & 14 & 34.1 & 13,14 & $13(s)$ \\
\hline 3.10 & $1 \mathrm{H}, \mathrm{ddd}$ & $\begin{array}{l}10.8,7.5 \\
5.0\end{array}$ & 6 & 5,14 & 46.2 & 5,14 & \\
\hline $\begin{array}{l}3.13- \\
3.18\end{array}$ & $1 \mathrm{H}, \mathrm{m}$ & & $5^{\prime}$ & & 36.5 & 6,14 & \\
\hline 3.78 & $3 \mathrm{H}, \mathrm{s}$ & & 26 & & 55.5 & & \\
\hline 6.24 & $1 \mathrm{H}, \mathrm{d}$ & 7.3 & $\mathrm{Ar}$ & & & & \\
\hline 6.28 & $1 \mathrm{H}, \mathrm{d}$ & 9.2 & 13 & 14 & 66.0 & $6,14,15$ & $\begin{array}{l}5(\mathrm{~m}) \\
5^{\prime}(\mathrm{s})\end{array}$ \\
\hline 6.63 & $1 \mathrm{H}, \mathrm{dd}$ & $8.9,2.9$ & PMP & & & & \\
\hline 6.69 & $1 \mathrm{H}, \mathrm{dd}$ & $8.9,2.5$ & PMP & & & & \\
\hline 6.78 & $2 \mathrm{H}, \mathrm{m}$ & & $\mathrm{Ar}$ & & & & \\
\hline 6.86 & $1 \mathrm{H}, \mathrm{dd}$ & $8.8,2.9$ & PMP & & & & \\
\hline 6.95 & $1 \mathrm{H}, \mathrm{m}$ & & PMP & & & & \\
\hline 7.00 & $1 \mathrm{H}$, apt t & 7.4 & $\mathrm{Ar}$ & & & & \\
\hline $\begin{array}{l}7.15- \\
7.35\end{array}$ & $9 \mathrm{H}, \mathrm{m}$ & & $\mathrm{Ar}$ & & & & \\
\hline \multirow[t]{9}{*}{7.40} & $1 \mathrm{H}, \mathrm{d}$ & 7.6 & $\mathrm{Ar}$ & & & & \\
\hline & & 289.4 & 20 & & 116.7 & & \\
\hline & & & 22 & & 127.8 & & \\
\hline & & & 16 & & 139.5 & & \\
\hline & & & 12 & & 139.6 & & \\
\hline & & & 4 & & 139.8 & & \\
\hline & & & 7 & & 145.7 & & \\
\hline & & 34.7 & 21 & & 158.6 & & \\
\hline & & & 25 & & 160.1 & & \\
\hline
\end{tabular}




\section{Structural elucidation of major diastereoisomer of $40 \mathrm{~d}$}

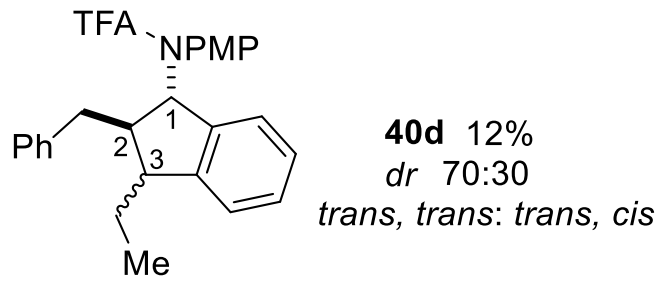

Analysis of the stereochemistry by NOESY interactions gave correlation between protons $\mathrm{H}_{1}-\mathrm{H}_{2}(w)$, $\mathrm{H}_{2}-\mathrm{H}_{3}(w)$ and $\mathrm{H}_{1}-\mathrm{H}_{3}(\mathrm{~s})$ suggesting a trans, trans-orientation. Analysis of ${ }^{3} \mathrm{JHH}_{\mathrm{HH}}$ small couplings ${ }^{3} \mathrm{~J}_{\mathrm{H} 1 \mathrm{H} 2}=7.6$, ${ }^{3} \mathrm{~J}_{\mathrm{H} 2 \mathrm{H} 3}=\sim 7.1 \mathrm{~Hz}$, in the range of observed values for cis-dialkyl substituted indanes. However the similar values across $\mathrm{H}_{1}-\mathrm{H}_{2}, \mathrm{H}_{2}-\mathrm{H}_{3}$ suggest a trans, trans-environment due to the similar ${ }^{3} \mathrm{~J}_{\mathrm{HH}}$ values suggesting a similar dihedral angle across both bonds. The chemical shift of the $\mathrm{CHN}$ proton $\left(\mathrm{H}_{1}, \delta 6.30 \mathrm{ppm}\right)$ is also close to those of $40 \mathrm{a}(\delta 6.28$ and $6.24 \mathrm{ppm})$ and $40 \mathrm{~b}(\delta 6.31$ and $6.28 \mathrm{ppm})$ suggesting a similar trans $-\mathrm{H}_{1} \mathrm{H}_{2}$ environment. The relative stereochemistry was therefore tentatively assigned as trans, trans- due to the consideration of all three pieces of data.

X-ray crystallography data. The single crystal X-ray diffractometer is equipped with an Atlas CCD Detector. All data sets collected at $150 \mathrm{~K}$ using CuKa radiation $(\lambda=1.54184 \AA$ ). The data were acquired and processed using the CrysAlis ${ }^{\text {Pro }}$ program. ${ }^{298}$ The datasets were corrected for Lorentz and polarization effects. Structure solution and refinement were accomplished using SHELXS-97 and SHELXL-97, ${ }^{299}$ respectively. The crystal structures were solved using direct methods. All nonhydrogen atoms were refined anisotropically, while hydrogen atoms associated with carbon and oxygen atoms were refined isotropically in geometrically constrained positions. Hydrogen atoms affiliated with nitrogen atoms were refined isotropically in positions identified in the difference Fourier map. Crystallographic and refinement parameters for crystal structures in section 4.4.2.

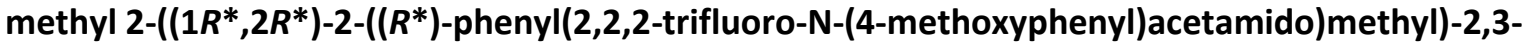
dihydro-1H-inden-1-yl)acetate (32a)
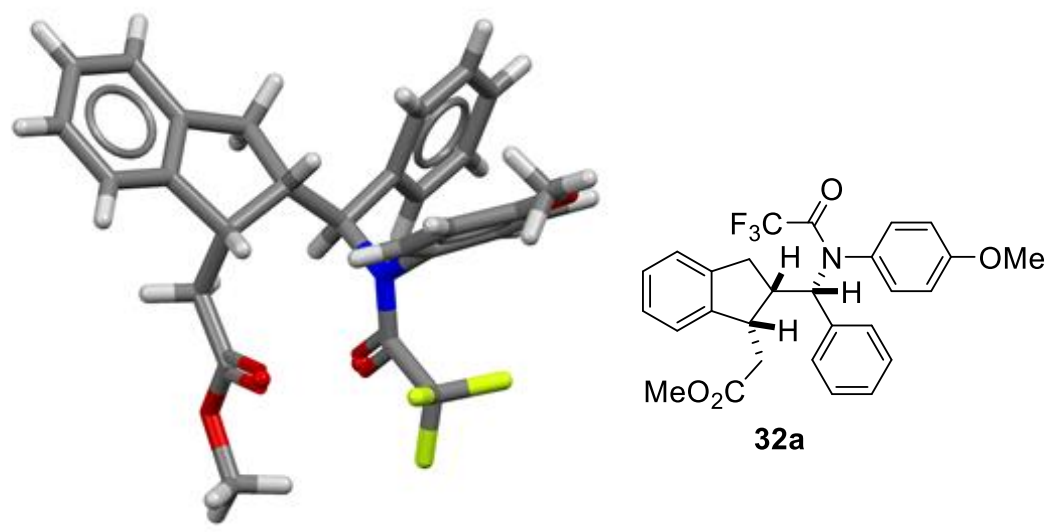


\begin{tabular}{|c|c|c|}
\hline chemical formula & $\mathrm{C}_{28} \mathrm{H}_{26} \mathrm{~F}_{3} \mathrm{NO}_{4}$ & \\
\hline$M_{\mathrm{r}} / \mathrm{g} \mathrm{mol}^{-1}$ & 497.50 & \\
\hline crystal system & monoclinic & \\
\hline space group & $P 2_{1} / c$ & \\
\hline \multirow[t]{3}{*}{ Unit cell dimensions } & $a=15.1930(3) \AA$ & $\alpha=90^{\circ}$ \\
\hline & $b=13.0292(2) \AA$ & $B=110.560(3)^{\circ}$ \\
\hline & $c=12.8223(3) \AA$ & $\gamma=90^{\circ}$ \\
\hline Volume & 2376.54(9) $\AA^{3}$ & \\
\hline Z & 4 & \\
\hline Density (calculated) & $1.39 \mathrm{~cm}^{-3}$ & \\
\hline$F(000)$ & 1040 & \\
\hline$\mu\left(\mathrm{Cu} K_{\alpha}\right)$ & $0.912 \mathrm{~mm}^{-1}$ & \\
\hline \multirow[t]{2}{*}{$T / K$} & $150(2)$ & \\
\hline & $-18 \rightarrow 18$ & \\
\hline \multirow[t]{2}{*}{ index range } & $-15 \rightarrow 15$ & \\
\hline & $-15 \rightarrow 10$ & \\
\hline collected reflections & 34570 & \\
\hline unique reflections & 4198 & \\
\hline$R_{\text {int }}$ & 0.1108 & \\
\hline reflections with $I>2 \sigma(I)$ & 3452 & \\
\hline no. parameters & 325 & \\
\hline$R(F), F>2 \sigma(F)$ & 0.0426 & \\
\hline$w R\left(F^{2}\right), F>2 \sigma(F)$ & 0.11 & \\
\hline$R(F)$, all data & 0.0529 & \\
\hline$w R\left(F^{2}\right)$, all data & 0.1171 & \\
\hline$\Delta_{r}(\min .$, max. $)$ e $\AA^{-3}$ & $-0.225,0.238$ & \\
\hline
\end{tabular}

(Z)-2,2,2-trifluoro- $N$-(4-methoxyphenyl)-N-((1-(phenyl(trimethylsilyl)methylene)-2,3-dihydro-1Hinden-2-yl)methyl)acetamide (35a)
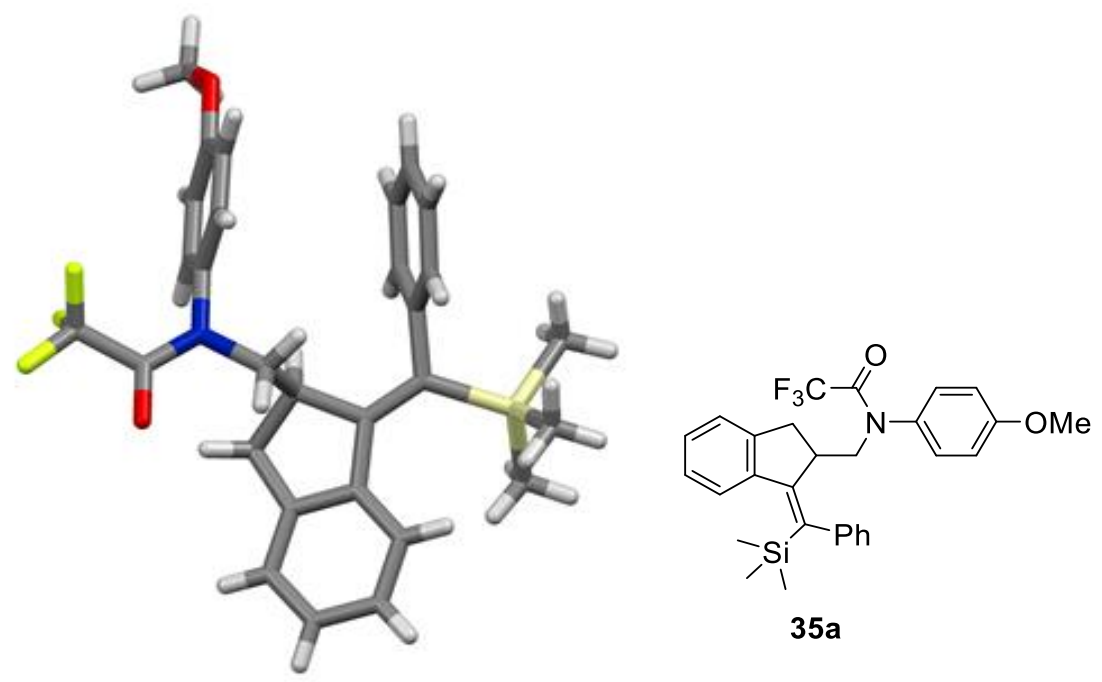

chemical formula $\mathrm{Mr}_{\mathrm{r}} / \mathrm{g} \mathrm{mol}^{-1}$

$\mathrm{C}_{29} \mathrm{H}_{30} \mathrm{~F}_{3} \mathrm{NO}_{2} \mathrm{Si}$ 509.64 


$\begin{array}{lll}\text { crystal system } & \text { triclinic } & \\ \text { space group } & P-1 & \alpha=115.009(4)^{\circ} \\ \text { Unit cell dimensions } & \mathrm{a}=10.1262(3) \AA & \beta=96.041(3)^{\circ} \\ & \mathrm{b}=12.1318(4) \AA & \gamma=104.045(3)^{\circ} \\ \text { Volume } & c=12.5425(4) \AA & \\ \text { Z } & 1316.22(8) \AA^{3} & \\ \text { Density (calculated) } & 2 & \\ F(000) & 1.226 \mathrm{~cm}^{-3} & \\ \mu\left(\text { Cu } K_{\alpha}\right) & 511 & \\ T / K & 1.162 \mathrm{~mm}^{-1} & \\ \text { index range } & 150(2) & \\ & -12 \rightarrow 12 & \\ \text { collected reflections } & -13 \rightarrow 14 & \\ \text { unique reflections } & -14 \rightarrow 14 & \\ R_{\text {int }} & 18582 & \\ \text { reflections with } I>2 \sigma(I) & 4623 & \\ \text { no. parameters } & 0.0474 & \\ R(F), F>2 \sigma(F) & 3884 & \\ w R\left(F^{2}\right), F>2 \sigma(F) & 0.0638 & \\ R(F), \text { all data } & 0.1751 & \\ w R\left(F^{2}\right) \text {, all data } & 0.0814 & \\ \Delta \mathrm{r}(\text { min., max. }) \text { e } \AA^{-3} & 0.1853 & \\ & -0.378,1.622\end{array}$

